\title{
Dependence of superconducting properties on the size and shape of a nanoscale superconductor: From nanowire to film
}

\author{
M. D. Croitoru, ${ }^{1}$ A. A. Shanenko, ${ }^{2,3}$ and F. M. Peeters ${ }^{2}$ \\ ${ }^{1}$ EMAT, Departement Fysica, Universiteit Antwerpen, Groenenborgerlaan 171, B-2020 Antwerpen, Belgium \\ ${ }^{2}$ TGM, Departement Fysica, Universiteit Antwerpen, Groenenborgerlaan 171, B-2020 Antwerpen, Belgium \\ ${ }^{3}$ Bogoliubov Laboratory of Theoretical Physics, Joint Institute for Nuclear Research, 141980 Dubna, Russia
}

(Received 22 December 2006; revised manuscript received 31 May 2007; published 18 July 2007)

\begin{abstract}
We analyze the dependence of the basic superconducting quantities - the order parameter, energy gap, and critical temperature - on the size and shape of a nanoscale superconductor in the clean limit. The Bogoliubov-de Gennes equations are solved numerically for a metallic nanowire with rectangular cross section. This makes it possible to vary the confining geometry, passing from a nanowire to a nanofilm and testing the sensitivity of the basic superconductive characteristics on the transverse size and shape of the nanowire. Strong size and shape superconducting resonances are found that depend on the geometry of the sample.
\end{abstract}

DOI: 10.1103/PhysRevB.76.024511

PACS number(s): $74.78 . \mathrm{Na}$

\section{INTRODUCTION}

Downscaling of superconducting device components as well as increasing the critical temperature and current are key challenges of nanoscale superconductivity. Thorough simulations that capture quantum-confinement effects in these devices will be essential to understand further experimental work. Despite intensive research on the superconductive properties of various materials and structures, there are still several problems hindering the application of such devices. Most important concerns the control of the size and shape of the carrier confinement that favors the enhancement of superconductivity and leads to an oscillatory behavior of the superconducting properties. Therefore, the study of superconductivity in nanostructures, such as nanowires, which have the possibility of tuning their characteristics by changing their cross section, is of fundamental interest.

Recent advances in fabrication technology of nanosized structures can make breakthroughs in the fundamental question about the size and shape dependences of the properties of nanoscale superconductors. ${ }^{1-12}$ Various techniques for fabricating nanoscale superconducting devices have recently been developed, amongst them methods based on extreme ultraviolet and electron-beam lithography. For example, in Ref. 11, it was shown that the latter method supplemented by ion-beam sputtering allows one to reduce the nanowire width down to $10 \mathrm{~nm}$.

Quantum-size effects are expected to strongly influence the physical (and superconducting) properties of highly crystalline nanoscale metallic structures. Several theoretical works have been devoted to the study of such effects in superconducting nanofilms. In particular, already more than 40 years ago, Blatt and Thompson investigated the multiband BCS model for a superconducting slab and found an oscillatory spikelike dependence of the energy-gap parameter on film thickness. ${ }^{13}$ These spikes are called size superconducting resonances. Recently, a cylindrical nanowire was investigated in Ref. 14 by means of the Bogoliubov-de Gennes $(\mathrm{BdG})$ equations. The superconducting order parameter was found to show significant spatial variations in the transverse direction due to quantum confinement for wire widths below the bulk coherence length. In the opposite case of large wire diameters, the Ginzburg-Landau approximation is recovered. Very recently, ${ }^{15,16}$ the critical temperature $T_{c}$ of a cylindrical superconducting nanowire was studied by solving numerically the BdG equations. A sequence of size-dependent resonances was found in $T_{c}$ when changing the nanowire width. A detailed comparison ${ }^{16}$ with experiment showed that such size-dependent resonances are responsible for the widthdependent increase in the superconducting temperature found in recent experiments on $\mathrm{Al}$ and $\mathrm{Sn}$ nanowires. ${ }^{10-12}$

In the present paper, we investigate the dependence of the basic superconductive quantities on the confining geometry (i.e., on the size and shape of a nanoscale superconductor). For this purpose, we numerically solve the BdG equations for a clean metallic nanowire with a rectangular cross section uniform over the whole wire length. This choice allows us to study the sensitivity of the superconducting properties on the specimen geometry when passing from a nanowire to a nanofilm. In particular, in addition to the size superconducting resonances, we investigate quantum superconducting resonances appearing when the shape of a nanoscale specimen is varied (shape-dependent superconducting resonances). The present paper is a generalization of our previous results on cylindrical nanowires ${ }^{15,16}$ and nanofilms ${ }^{17}$ to a rectangular shaped wire. Our previous results are special limits of the case considered here.

The paper is organized as follows. In Sec. II, we discuss the $\mathrm{BdG}$ equations for a nanowire with rectangular cross section. Details of our numerical method to solve these equations self-consistently are presented in this section. Our numerical results and the physical interpretation are given in Sec. III. The conclusions are presented in Sec. IV.

\section{MODEL}

In order to study quantum-geometry effects, we investigate a clean superconducting nanowire with rectangular cross section (transverse dimensions $L_{x}$ and $L_{y}$ ). Note that when calculating the critical superconducting temperature (and the order parameter), a nanowire can be considered as being in the clean limit if the electron mean free path is 
about or larger than the nanowire transverse dimensions (such nanowires are now attainable in experiments ${ }^{10,11}$ ). In this case, nonmagnetic impurities influence only the electron motion parallel to the nanowire. Proceeding in the spirit of the well-known Anderson theorem, we can conclude that $T_{c}$ is not much influenced by nonmagnetic impurities in such a nanowire. Furthermore, we take the electron band-mass isotropic (i.e., $m_{x}=m_{y}=m_{z}=m$ ). As known since the classical papers by Gor'kov ${ }^{18}$ and Bogoliubov, ${ }^{19}$ the superconducting order parameter can be seen as the wave function governing the center-of-mass motion of the Cooper pairs. Such quantum-confinement effects cannot be studied within the Ginzburg-Landau theory, and a more complex approach as, e.g., based on the $\mathrm{BdG}$ equations ${ }^{20}$ is needed. In the absence of a magnetic field, the order parameter can be chosen as a real quantity, and the BdG equations are written as

$$
\left[\hat{H}_{e} \sigma_{z}+\Delta \sigma_{x}\right] \psi_{i}=E_{i} \psi_{i},
$$

where

$$
\psi_{i}(\mathbf{r})=\left(\begin{array}{c}
u_{i}(\mathbf{r}) \\
v_{i}(\mathbf{r})
\end{array}\right)
$$

is the two-component (particle and hole) wave function corresponding to the quasiparticle (bogolon) energy $E_{i} ; \sigma_{x}$ and $\sigma_{z}$ are the Pauli matrices acting in the particle-hole space; the single-electron Hamiltonian $\hat{H}_{e}$ reads

$$
\hat{H}_{e}=-\frac{\hbar^{2}}{2 m} \nabla^{2}+V(\mathbf{r})-\mu,
$$

with $\mu$ the chemical potential and $V(\mathbf{r})=V_{B} \theta(|x|$ $\left.-L_{x} / 2\right) \theta\left(|y|-L_{y} / 2\right)$ the confining potential $\left(V_{B}\right.$ is the barrier parameter assumed to be infinite in the present paper, and $\theta(x)$ is the Heaviside step function). For a rectangular nanowire $i=\left\{i_{x}, i_{y}, k_{z}\right\}$, where $i_{x}$ and $i_{y}$ label the subbands appearing due to the size quantization of the electron motion in the transverse $x$ and $y$ directions, and $k_{z}$ is the wave vector of the quasifree electron motion along the $z$ direction.

In order to find the quasiparticle energy spectrum $E_{i}$ and corresponding wave functions $u_{i}(\mathbf{r})$ and $v_{i}(\mathbf{r})$, we need to solve the BdG equations self-consistently together with the relation

$$
\Delta(\mathbf{r})=g \sum_{i \in \mathcal{C}} u_{i}(\mathbf{r}) v_{i}^{*}(\mathbf{r})\left[1-2 f\left(E_{i}\right)\right],
$$

where $g$ is the coupling constant and $f\left(E_{i}\right)=1 /\left(e^{\beta E_{i}}+1\right)$ is the Fermi function $\left[\beta=1 /\left(k_{B} T\right)\right.$ with $T$ the temperature and $k_{B}$ the Bolzmann constant]. It is important that the summation in Eq. (3) is over all the eigenstates that have positive quasiparticle energy $E_{i}$ and the single-electron energy

$$
\xi_{i}=\int d^{3} r\left[u_{i}^{*}(\mathbf{r}) \hat{H}_{e} u_{i}(\mathbf{r})+v_{i}^{*}(\mathbf{r}) \hat{H}_{e} v_{i}(\mathbf{r})\right]
$$

within the Debye "window" $\left[-\hbar \omega_{D}, \hbar \omega_{D}\right]\left(\omega_{D}\right.$ is the Debye frequency). Below, we indicate by $\mathcal{R}$ the full set of quantum numbers corresponding to all the eigenstates with positive energy, whereas $\mathcal{C}$ denotes the subset of the eigenstates with $\xi_{i \in \mathcal{C}} \in\left[-\hbar \omega_{D}, \hbar \omega_{D}\right]$. For a given mean electron density $n$ the chemical potential $\mu$ is determined by

$$
n=\frac{2}{V} \sum_{i \in \mathcal{R}} \int d^{3} r\left\{\left|u_{i}(\mathbf{r})\right|^{2} f\left(E_{i}\right)+\left|v_{i}(\mathbf{r})\right|^{2}\left[1-f\left(E_{i}\right)\right]\right\},
$$

with $V=L_{x} L_{y} L_{z}$. Along the $z$ direction, we introduce a unit cell of length $L_{z}$ which is repeated periodically. Due to the quantum confinement in the transverse directions, we have to impose the following boundary conditions:

$$
\left.u_{i}(\mathbf{r})\right|_{\mathbf{r} \in S}=\left.v_{i}(\mathbf{r})\right|_{\mathbf{r} \in S}=0
$$

on the wire surface, while in the longitudinal direction periodic boundary conditions are used. Equations (1)-(6) are the basic formulas needed to obtain a self-consistent solution.

In order to numerically solve the BdG equations (1), we expand the two-component wave function $\psi_{i}(\mathbf{r})$ as

$$
\left(\begin{array}{c}
u_{i}(\mathbf{r}) \\
v_{i}(\mathbf{r})
\end{array}\right)=\frac{e^{\imath k_{z} z}}{\sqrt{L_{z}}} \sum_{j_{x}, j_{y}>0} \varphi_{j_{x} j_{y}}(x, y)\left(\begin{array}{c}
u_{j_{x} j_{y}}^{i} \\
v_{j_{x} j_{y}}^{i}
\end{array}\right),
$$

in terms of the quantum well states

$$
\varphi_{j_{x} j_{y}}(x, y)=\frac{2}{\sqrt{L_{x} L_{y}}} \sin \left(\pi j_{x} \frac{x}{L_{x}}\right) \sin \left(\pi j_{y} \frac{y}{L_{y}}\right) .
$$

Now we can make a transformation from the twodimensional to one-dimensional notation of the indices, using the mapping rule $j\left(j_{x}, j_{y}\right)=j_{y}+j_{x} N_{y}$, where $N_{y}$ is the total number of eigenstates associated with the $y$ direction that are included in our numerical calculations. After inserting Eq. (7) into the BdG equations, we obtain the following equations for the expansion coefficients $\left(j=\left\{j_{x}, j_{y}, k_{z}\right\}\right)$ :

$$
\begin{aligned}
& \left(T_{j}^{i}-E_{i}\right) u_{j}^{i}+\sum_{j^{\prime}} \Delta_{j j^{\prime}} v_{j^{\prime}}^{i}=0, \\
& \left(E_{i}+T_{j}^{i}\right) v_{j}^{i}-\sum_{j^{\prime}} \Delta_{j j^{\prime}} u_{j^{\prime}}^{i}=0,
\end{aligned}
$$

where $\left[j=j\left(j_{x}, j_{y}\right)\right]$

$$
T_{j}^{i}=\frac{\hbar^{2}}{2 m}\left(\frac{\pi^{2} j_{x}^{2}}{L_{x}^{2}}+\frac{\pi^{2} j_{y}^{2}}{L_{y}^{2}}+k_{z}^{2}\right)-\mu
$$

and

$$
\Delta_{j j^{\prime}}=\int d x d y \varphi_{j_{x} j_{y}}(x, y) \Delta(x, y) \varphi_{j_{x}^{\prime} j_{y}^{\prime}}(x, y) .
$$

We remark that in the case of interest, the superconducting order parameter depends only on the transverse variables, i.e., $\Delta(\mathbf{r})=\Delta(x, y)$. Then, Eqs. (9) are readily converted into a matrix form so that the eigenvalues and eigenfunctions of the problem can be found by means of diagonalizing the relevant matrix. The criterion of selecting quasiparticle states in Eq. (3) $\left(\xi_{i} \in\left[-\hbar \omega_{D}, \hbar \omega_{D}\right]\right)$ dictates that one should at least include those $j_{x}$ and $j_{y}$ for which $T_{j=j\left(j_{x}, j_{y}\right)}^{i} \in\left[-\hbar \omega_{D}, \hbar \omega_{D}\right]$. Numerical analysis shows that such an approximation leads, as a rule, to results with accuracy of a few percent. 
TABLE I. Debye temperature $\hbar \omega_{D} / k_{B}$ and bulk density of states $N(0)$ multiplied by the interaction constant $g$.

\begin{tabular}{lccc}
\hline \hline & $\mathrm{Al}$ & $\mathrm{Sn}$ & $\mathrm{Pb}$ \\
\hline$\hbar \omega_{D} / k_{B}(\mathrm{~K})$ & 375 & 195 & 96 \\
$g N(0)$ & 0.18 & 0.25 & 0.39 \\
\hline \hline
\end{tabular}

\section{RESULTS AND DISCUSSION}

In our numerical investigations, we restrict ourselves to $\mathrm{Al}, \mathrm{Sn}$, and $\mathrm{Pb}$ nanowires whose bulk material parameters are given in Table I (data are taken from Refs. 20-22).

In the present model, the bulk density of single-electron states per unit volume and per spin projection reads

$$
N(0)=\frac{m k_{F}}{2 \pi^{2} \hbar^{2}}
$$

with $k_{F}$ the three-dimensional Fermi wave vector. To simulate an infinite nanowire we took the length $L_{z}=500 \mathrm{~nm}$ $\gg \lambda_{F}=2 \pi / k_{F}$ (the Fermi wavelength) for the periodic boundary in the $z$ direction. Quantitative description of the recently observed quantum-size oscillations in the critical temperature of superconducting nanofilms ${ }^{1}$ as well as a detailed understanding of the size-dependent variations in the work function, the surface energy and the thermal stability ${ }^{23-27}$ require the knowledge of the crystal band structure in the presence of quantum confinement. As shown in Ref. 28, this procedure, to a certain extent, can be avoided by using the band-mass approximation together with a change of the reference point in the band structure. This can be realized by the introduction of an effective Fermi level. ${ }^{17}$ The effective Fermi levels for materials in question are taken as follows. For a $\mathrm{Pb}$ nanowire, we use $\mu_{\text {bulk }}=1.42 \mathrm{eV}$ which follows from the recent results found in Ref. 4. For Sn, we put $\mu_{\text {bulk }}=2.27 \mathrm{eV}$ in agreement with Ref. 5. For Al, we employ $\mu_{\text {bulk }}=0.9 \mathrm{eV}$, which provides a good agreement with the very recent data on the size-dependent enhancement of $T_{c}$ in aluminum superconducting nanowires.

Figure 1 illustrates the resonant structure appearing in $T_{c} / T_{c \text {,bulk }}$ in $\mathrm{Al}, \mathrm{Sn}$, and $\mathrm{Pb}$ nanowires. The relative transition temperature exhibits pronounced resonances that result from peaks appearing in the mean density of states (due to the Van Hove singularities). ${ }^{15,16}$ It is important that the critical temperature increases well above its bulk value at a resonant point but, then, drops down (often below $T_{c \text {,bulk }}$ ) until a sequent resonance comes into play. However, the resonance enhancements are as a rule much more pronounced than the drops between two neighboring resonances (with respect to $\left.T_{c \text {, bulk }}\right)$. In addition, the larger the resonance magnitude, the larger the drop below $T_{c \text {, bulk }}$ between these resonances. As one can see from this figure, the resonances in $T_{c} / T_{c \text {,bulk }}$ are rather sensitive to the governing parameters $\omega_{D}, g$, and $\mu_{\text {bulk }}$. Comparing results for different materials, it is possible to see that the superconductive resonances weaken with increase of any of these parameters. As follows from Fig. 1, aluminium nanowires exhibit the most pronounced resonances. The resonance structure for tin is less significant due to the larger $\mu_{\text {bulk }}$ and $g$. Enhancement in the electron-phonon coupling in
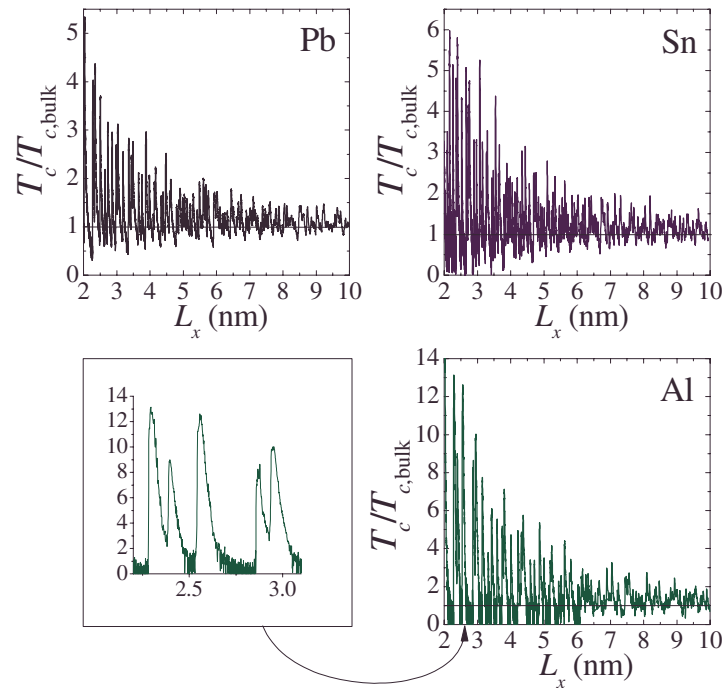

FIG. 1. (Color online) The $L_{x}$-dependent relative critical temperature $T_{c} / T_{c \text {, bulk }}$ for $\mathrm{Pb}, \mathrm{Sn}$, and $\mathrm{Al}$ square-cross-section nanowires using an effective Fermi level.

lead results in a considerable suppression of the multi-spike pattern for the $\mathrm{Pb}$ nanowire. Such dependencies are explained by the fact that the superconducting resonances result from a competition between quantum-confinement energy and superconducting "condensation" energy: the larger the condensation energy, the weaker the width-dependent resonances. Note that the obtained results are qualitatively (but not quantitatively) similar to those found for cylindrical $\mathrm{Al}$ and Sn nanowire. ${ }^{15,16}$ We remark that drops of the critical temperature below $T_{c \text {,bulk }}$ in Fig. 1 are somewhat enhanced with a decrease of the nanowire width. For example, for aluminum, $T_{c}$ can even decrease to zero when $L_{z}<6.5 \mathrm{~nm}$. Stress that these drops occur in nanowires with a cross section uniform over the whole nanowire length. In real samples, there are inevitable cross-section variations so that the critical temperature measured experimentally cannot be compared directly with $T_{c}$ presented in Fig. 1. The theoretical results obtained for a uniform nanowire should, therefore, be averaged over the particular cross-section variations, and such averaging will be influenced by the superconducting resonances. Indeed, as shown in Ref. 16, recent experimental data for Al (Refs. 10 and 11) and Sn (Ref. 12) nanowires follow the average trend of the size-dependent superconducting resonances calculated via the $\mathrm{BdG}$ equations for a cylindrical uniform nanowire. ${ }^{16}$

In the left lower panel of Fig. 1, we show the difference between the size-dependent resonances and the peaks resulting from the fluctuations of $N_{D}$ [the number of singleelectron states (for one spin projection) situated in the Debye window] due to a discrete structure of equispaced $z$ momenta (we use periodic boundary conditions for the electron motion in the $z$ direction). The former have large magnitudes $(\sim 10)$, the latter are small and play only a role in the regions between the resonances. The "undercoat" of these secondary peaks is washed out when the nanowire length $L_{z}$ increases above $2-4 \mu \mathrm{m}$. The number of single-electron states situated in the Debye window increases together with the nano- 

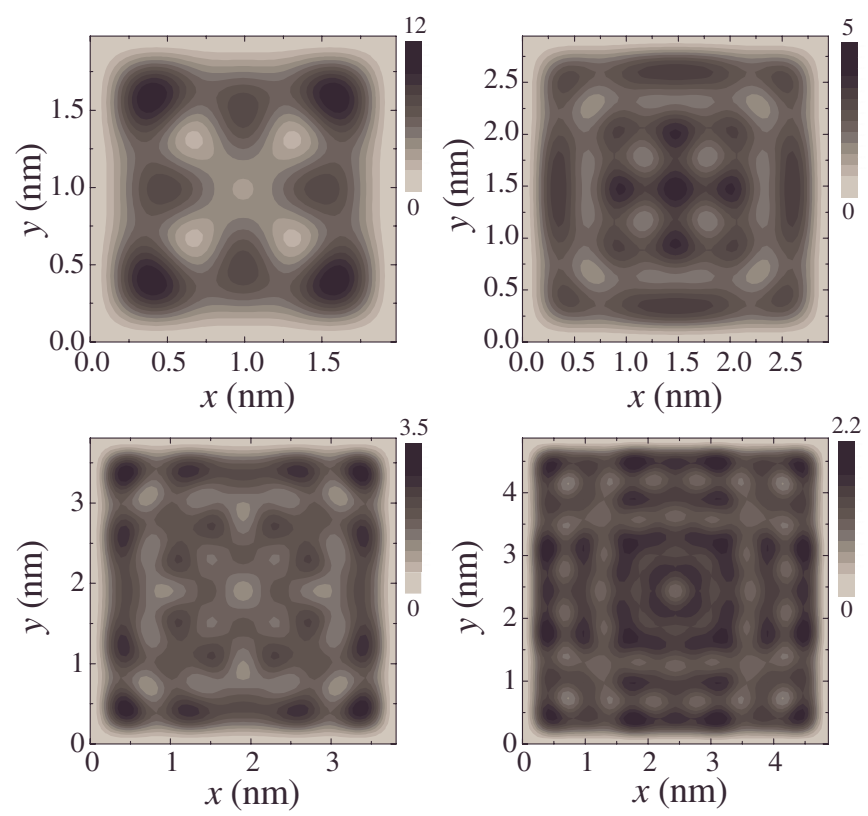

FIG. 2. (Color online) Contour plots for $\Delta(x, y)(\mathrm{meV})$ in an $\mathrm{Al}$ nanowire with square cross section. Results are shown for the resonant points $L_{x}=1.98,2.95,3.81$, and $4.87 \mathrm{~nm}$. The bulk gap $\Delta_{\text {bulk }}=0.25 \mathrm{meV}$.

wire width, which leads to a suppression of the relative $N_{D}$ fluctuations.

Quantum confinement also results in a nonuniform distribution of the pair condensate. In the presence of a superconducting resonance, this distribution shows striking patterns reflecting a complex interplay of the boundaries with areas of strongly enhanced order parameter. In Fig. 2, the contour plots of $\Delta(x, y)$ in an aluminum nanowire with square cross section are presented for the resonant points $L_{x}=1.98$, $2.95,3.81$, and $4.87 \mathrm{~nm}$. Notice that any superconductive resonance demonstrates its unique spatial structure which is never repeated at another resonant point. In the areas, where the superconducting order parameter is enhanced, it significantly exceeds the bulk gap $\Delta_{\text {bulk }}=1.27 \mathrm{meV}$. To go into more detail about the spatial variations of the superconducting order parameter, the "diagonal" function $\left.\Delta(x, y)\right|_{x=y}$ is plotted versus $L_{x}$ for the resonant point $L_{x}=2.95 \mathrm{~nm}$ (see the upper right panel of Fig. 2), and for the points $L_{x}$ $=2.98,3.0$, and $3.05 \mathrm{~nm}$ giving an idea about the decay of this resonance when we move away from the resonant point. The spatial distribution of the order parameter is strongly inhomogeneous in the presence of a superconducting resonance. However, the off-resonance superconducting condensate (see curve 4 in Fig. 3) is almost uniform in the central region of the sample. Near the edges, the diagonal order parameter $\left.\Delta(x, y)\right|_{x=y}$ rapidly varies, decreasing to zero on a scale of order $\lambda_{F} / 2$. Note that in Fig. 3, the superconducting order parameter is enhanced exactly in the center of the nanowire for the resonance curves. This is only by accident. In fact, a lot of different patterns of the spatial distribution of the resonant pair condensate exist. Some of them exhibit an enhancement in the center of the nanowire but there exist also opposite situations (see, for instance, the lower right

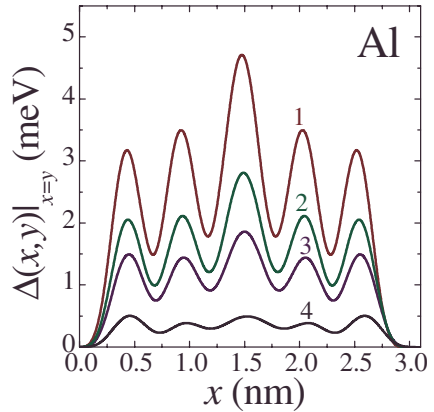

FIG. 3. (Color online) The diagonal order parameter $\left.\Delta(x, y)\right|_{x=y}$ in an aluminum nanowire with square cross section: curves 1-4, $L_{x}=2.95$ (a resonant point), 2.98, 3.0, and $3.05 \mathrm{~nm}$.

panel in Fig. 2). The resonant patterns given in Fig. 2 exhibit a clear $C_{4}$ symmetry related to the square shape of the cross section. However, when the nanowire width exceeds $\lambda_{F} / 2$ by more than an order of magnitude, the spatial distribution of the order parameter in the center begins "to forget" about the symmetry of the boundaries. In particular, in the center of the lower right panel of Fig. 2, there are signatures of a starting circular distribution typical for a cylindrical nanowire.

Heretofore, we discussed the numerical results of the BdG equations in a nanowire with square cross section, where we went into detail about the size-dependent superconducting resonances. However, the superconductive properties of a nanoscale specimen exhibit also an interesting dependence on its shape. Here, this dependence is investigated for a nanowire with rectangular cross section. Figure 4 shows our results for the critical temperature calculated for $\mathrm{Pb}$ nanowires with different ratios $L_{y} / L_{x}$ (the effective Fermi level $\mu_{\text {bulk }}=0.927 \mathrm{meV}$ is used). The results are plotted versus the square root of the cross section $\sigma=L_{x} \times L_{y}$ (with steps of $0.002 \mathrm{~nm}$ ). The most important point about the data in Fig. 4 is that the size-dependent superconducting resonances start
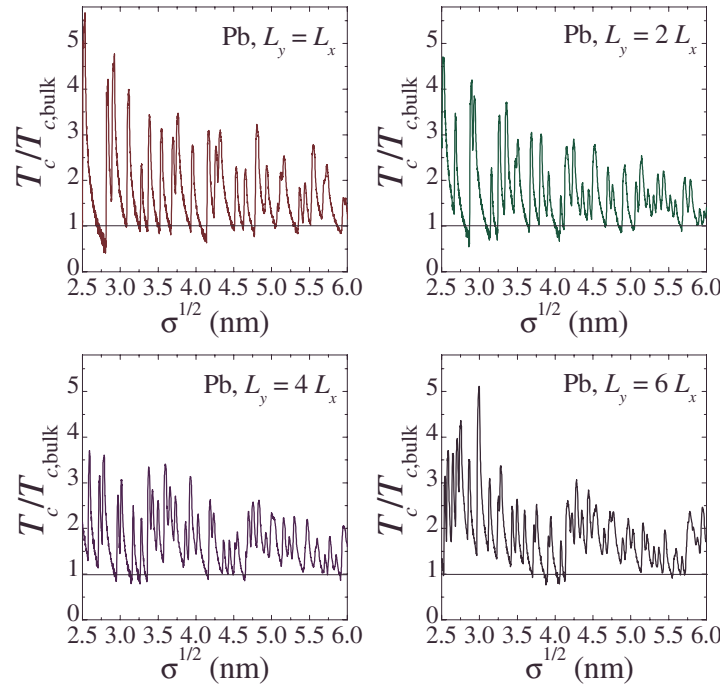

FIG. 4. (Color online) The shape-dependent superconducting temperature as a function of the square root of the cross section $\sigma$ $=L_{x} L_{y}$ for a set of rectangular $\mathrm{Pb}$ nanowires with $L_{y}=L_{x}, 2 L_{x}, 4 L_{x}$, and $6 L_{x}$. 


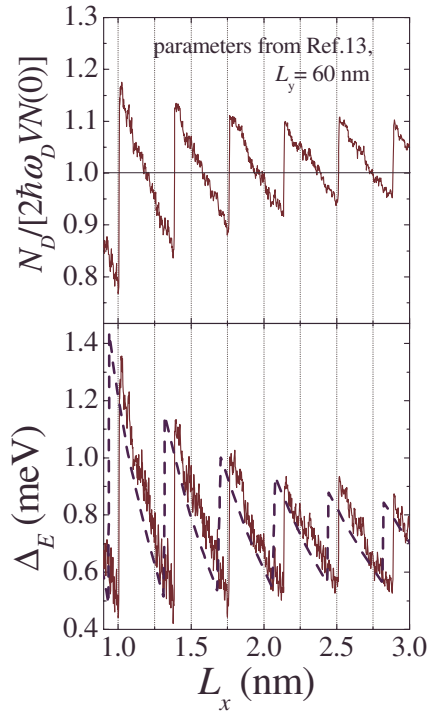

FIG. 5. (Color online) The relative mean density of states $N_{D} /\left[2 \hbar \omega_{D} V N(0)\right]$ (the upper panel) and the energy gap $\Delta_{E}$ (the lower panel) versus the width $L_{x}$ for a rectangular nanowire with $L_{y}=60 \mathrm{~nm}$. The dashed line in the lower panel represents the results of Blatt and Thompson (see Ref. 13).

to group for $L_{y}=4 L_{x}$ and $L_{y}=6 L_{x}$. There is no change in the density of the resonances per unit effective size $\sigma^{1 / 2}$. The grouping occurs due to a significant change in the drops of $T_{c}$ between the superconducting resonances. Indeed, when a resonance decays with an increase in $\sigma^{1 / 2}$ for the case $L_{y}$ $=L_{x}$, the critical temperature falls typically down to its bulk value or somewhat below. On the contrary, in the panel $L_{y}$ $=6 L_{x}$, we have a different pattern with a clear combination of the two structures: fast variations (size resonances associated with the $y$ direction) are superimposed on the relatively slow oscillations (they are due to quantum confinement in the $x$ direction). These slow oscillations are nucleating nanofilm resonances. A sample with $L_{y}=6 L_{x}$ exhibits similarities with both a nanowire and a nanofilm, and this fact manifests itself in the presence of the two structures in the right lower panel of Fig. 4. It is surprising that signatures of nanofilm resonances appear even at $L_{y}=4 L_{x}$. Thus, we arrive at the important conclusion that quantum-size oscillations which are a property of nanoscale superconductors are accompanied by significant quantum-shape variations. Recall the reader that these results are for lead. The quantum-shape effect for tin and aluminum is even more profound.

When the ratio $L_{y} / L_{x}$ increases, the slowly oscillating curve mentioned in the lower right panel of Fig. 4 becomes clearer. The superimposed fast size-dependent oscillations due to quantization in the $y$ direction weaken and, finally, disappear. In Fig. 5, we present the mean density of states (the upper panel) and the energy gap (the lower panel) for a rectangular nanowire with $L_{y}=60 \mathrm{~nm}$ and $L_{x}=1-3 \mathrm{~nm}$ (the data are plotted with steps $\Delta L_{x}=0.002 \mathrm{~nm}$ ). In this case, the transverse ratio variations are $L_{y} / L_{x}=20-60$. The results were calculated for the parameters $\hbar \omega_{D} / k_{B}=100 \mathrm{~K}, g N(0)$ $=0.3$, and $\mu_{\text {bulk }}=2.69 \mathrm{eV}$ taken from the paper by Blatt and Thompson, ${ }^{13}$ in order to compare our data (the solid line in the lower panel) with the results of Ref. 13 found for a thin

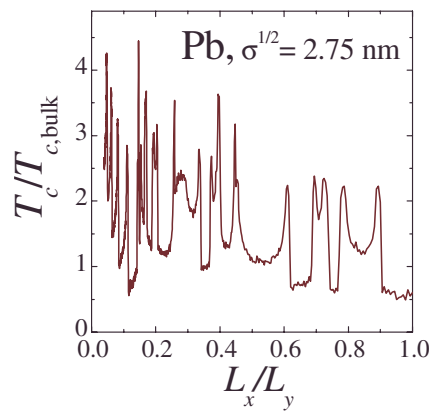

FIG. 6. (Color online) Shape-dependent superconducting reso-

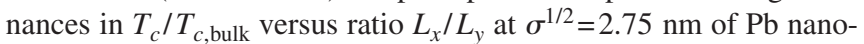
wire with rectangular cross section.

superconducting slab (the dashed line in the lower panel). The small shift between the solid and dashed curves in the lower panel is due to the fact that the calculations performed by Blatt and Thompson do not take into account change in $\mu$ due to quantum confinement. Another difference concerns the surviving small fast oscillations present in the numerical results for the rectangular nanowire. In addition, the amplitudes of the slow oscillations of the solid curve in the lower panel are somewhat (within 10\%) different as compared to the dashed curve. This is because the results by Blatt and Thompson were calculated within the multiband BCS model but not within the more accurate $\mathrm{BdG}$ equations.

The nanowire-to-nanofilm transformation is also illustrated by Fig. 6, where the relative critical temperature in $\mathrm{Pb}$ nanowire with rectangular cross section is plotted versus the ratio $L_{x} / L_{y}$ for $\sigma^{1 / 2}=2.75 \mathrm{~nm}$. When $L_{x} / L_{y}$ is about 1 (a nearly square nanowire), we observe a sequence of shapedependent superconducting resonances with a camel backlike profile. This camel backlike structure appears due to the correlated passages through the Fermi surface of the bottoms of the two electron subbands. One subband, associated with the quantization of the electron motion in the $y$ direction, moves down in energy. When its bottom passes through the Fermi surface, we get the right hump of a shape-dependent resonance. The other subband, arising due to quantization in the $x$ direction, moves up in energy, and this movement results in the left hump of a shape superconducting resonance. The linear density of the $T_{c}$ enhancements associated with the $y$ direction increases with a decrease in shape parameter $L_{x} / L_{y}$. The linear density of the $T_{c}$ enhancements due to quantization in the $x$ direction also increases but much weaker. Hence, near the point $L_{x} / L_{y}=1$ the shape superconducting resonances have the simple two-hump structure. But for $L_{x} / L_{y} \approx 0.2-0.4$, shape resonances become complex, including more and more enhancements of $T_{c}$ formed due to quantum confinement in the $y$ direction. Finally, for $L_{x} / L_{y}$ $\ll 1$, we arrive at the quasinanofilm regime similar to that of the right lower panel of Fig. 4: fast quantum oscillations are superimposed on the slowly varying curve. Note that the regime $L_{x} / L_{y} \rightarrow 0$ is unphysical for any given value of the cross section $\sigma$ because in this case we arrive at a nanofilm with thickness less than one monolayer.

We remark that one can simplify the calculational procedure for the rectangular nanowire with a large transverse ratio $L_{y} / L_{x}$ by introducing the periodic boundary conditions 
in the $y$ direction. This will lead to the picture very similar to Fig. 5: we again have a slowly varying curve with superimposed fast oscillations for the mean density of states and energy gap. However, now such oscillations are the undercoat (see Fig. 2) appearing due to the equispaced discrete structure of $z$ momenta. The positions of the undercoat peaks will be different with respect to the small surviving resonances presented in Fig. 5 but their amplitudes will be similar.

\section{CONCLUSION}

In conclusion, quantum confinement is the cornerstone mechanism governing nanoscale superconductivity. Based on a numerical solution of the Bogoliubov-de Gennes equations, we investigated how the superconducting properties of a nanoscale specimen depend on the confining geometry. With this aim, we solved these equations for the case of a clean metallic nanowire with rectangular cross section $\sigma$ $=L_{x} L_{y}$ uniform over the wire length. When passing from a nanowire to a nanofilm by changing the transverse dimensions, we were able to explore the sensitivity of the basic superconducting properties on the size and shape of the nanowire.

For the case $L_{y} / L_{x}=1$ (a nanowire with square cross section), we investigated the width-dependent superconducting resonances responsible for the remarkable quantum-size os- cillations of the superconducting properties typical for nanoscale superconductivity and first calculated by Blatt and Thompson for a thin superconducting slab. ${ }^{13}$ Recently, such oscillations were observed in experiments with $\mathrm{Pb}(111)$ nanoterraces grown on a silicon substrate. ${ }^{1}$ We showed that the superconducting order parameter is enhanced at a resonant point, and its spatial distribution exhibits a distinguished nonuniform pattern reflecting the symmetry of the sample. Such a pattern changes from resonance to resonance, and there is no simple "thumb rule" for predicting the paircondensate distribution.

For $L_{y} / L_{x}>1$ (passing from the nanowire to the nanofilm regime), we investigated how the transverse shape can influence the superconducting characteristics, comparing the data calculated for different ratios $L_{y} / L_{x}$ but for the same cross section $\sigma$. This allowed us to conclude that quantum-size oscillations are accompanied by quantum-shape variations in all the superconducting properties. We believe that the nontrivial interplay of the quantum-size and quantum-shape effects can be a possible avenue toward an optimal design of future nanostructured superconducting devices.

\section{ACKNOWLEDGMENTS}

This work was supported by the Flemish Science Foundation (FWO-Vl), the Belgian Science Policy (IAP), and BOF-TOP (University of Antwerp).
${ }^{1}$ Y. Guo, Y.-F. Zhang, X.-Y. Bao, T.-Z. Han, Z. Tang, L.-X. Zhang, W.-G. Zhu, E. G. Wang, Q. Niu, Z. Q. Qiu, J.-F. Jia, Z.-X. Zhao, and Q. K. Xue, Science 306, 1915 (2004).

${ }^{2}$ M. M. Özer, J. R. Thomson, and H. H. Weitering, Nat. Phys. 2, 173 (2006).

${ }^{3}$ X.-Y. Bao, Y.-F. Zhang, Y. Wang, J.-F. Jia, Q.-K. Xue, X. C. Xie, and Z.-X. Zhao, Phys. Rev. Lett. 95, 247005 (2005).

${ }^{4}$ Y.-F. Zhang, J.-F. Jia, T.-Z. Han, Z. Tang, Q.-T. Shen, Y. Guo, Z. Q. Qiu, and Q.-K. Xue, Phys. Rev. Lett. 95, 096802 (2005).

${ }^{5}$ B. G. Orr, H. M. Jaeger, and A. M. Goldman, Phys. Rev. Lett. 53, 2046 (1984).

${ }^{6}$ B. G. Orr, H. M. Jaeger, and A. M. Goldman, Phys. Rev. B 32, 7586 (1985).

${ }^{7}$ A. Bezryadin, C. N. Lau, and M. Tinkham, Nature (London) 404, 971 (2000).

${ }^{8}$ M. A. Skvortsov and M. V. Feigel'man, Phys. Rev. Lett. 95, 057002 (2005).

${ }^{9}$ M. Harmele, G. Rafael, M. P. A. Fisher, and P. M. Goldbart, Nat. Phys. 1, 117 (2005).

${ }^{10}$ M. Savolainen, V. Touboltsev, P. Koppinen, K.-P. Riikonen, and K. Arutyunov, Appl. Phys. A: Mater. Sci. Process. 79, 1769 (2004).

${ }^{11}$ M. Zgirski, K.-P. Riikonen, V. Touboltsev, and K. Arutyunov, Nano Lett. 5, 1029 (2005).

${ }^{12}$ M. Tian, J. Wang, J. S. Kurtz, Y. Liu, M. H. W. Chan, T. S. Mayer, and T. E. Mallouk, Phys. Rev. B 71, 104521 (2005).

${ }^{13}$ J. M. Blatt and C. J. Thompson, Phys. Rev. Lett. 10, 332 (1963).

${ }^{14}$ J. E. Han and V. H. Crespi, Phys. Rev. B 69, 214526 (2004).
${ }^{15}$ A. A. Shanenko and M. D. Croitoru, Phys. Rev. B 73, 012510 (2006).

${ }^{16}$ A. A. Shanenko, M. D. Croitoru, M. Zgirski, F. M. Peeters, and K. Arutyunov, Phys. Rev. B 74, 052502 (2006).

${ }^{17}$ A. A. Shanenko, M. D. Croitoru, and F. M. Peeters, Europhys. Lett. 76, 498 (2006); Phys. Rev. B 75, 014519 (2007).

${ }^{18}$ L. P. Gor'kov, Sov. Phys. JETP 7, 505 (1958).

${ }^{19}$ N. N. Bogoliubov, Sov. Phys. Usp. 67, 549 (1959).

${ }^{20} \mathrm{P}$. G. de Gennes, Superconductivity of Metals and Alloys (Bejamin, New York, 1966).

${ }^{21}$ A. L. Fetter and J. D. Walecka, Quantum Theory of ManyParticle Systems (Dover, New York, 2003).

${ }^{22}$ N. W. Ashcroft and N. D. Mermin, Solid State Physics (Holt, Rinehart and Winston, New York, 1976).

${ }^{23}$ A. R. Smith, K.-J. Chao, Q. Niu, and C. K. Shih, Science 273, 226 (1996).

${ }^{24}$ L. Gavioli, K. R. Kimberlin, M. C. Tringides, J. F. Wendelken, and Z. Zhang, Phys. Rev. Lett. 82, 129 (1999).

${ }^{25}$ K. Budde, E. Abram, V. Yeh, and M. C. Tringides, Phys. Rev. B 61, R10602 (2000); V. Yeh, L. Berbil-Bautista, C. Z. Wang, K. M. Ho, and M. C. Tringides, Phys. Rev. Lett. 85, 5158 (2000).

${ }^{26}$ M. Hupalo, S. Kremmer, V. Yeh, L. Berbil-Bautista, and M. C. Tringides, Surf. Sci. 493, 526 (2001); M. Hupalo and M. C. Tringides, Phys. Rev. B 65, 115406 (2002).

${ }^{27}$ W. B. Su, S. H. Chang, W. B. Jian, C. S. Chang, L. J. Chen, and T. T. Tsong, Phys. Rev. Lett. 86, 5116 (2001).

${ }^{28}$ C. M. Wei and M. Y. Chou, Phys. Rev. B 66, 233408 (2002). 